\title{
Ciliopathy variant burden and developmental delay in children with hypoplastic left heart syndrome
}

\author{
Gabrielle C. Geddes, MD1, Karl Stamm, MS², Michael Mitchell, MD², Kathleen A. Mussatto, PhD, RN² \\ and Aoy Tomita-Mitchell, $\mathrm{PhD}^{2}$
}

Purpose: To test the hypothesis that patients with hypoplastic left heart syndrome (HLHS) and developmental delay will have a higher average summative C-score in ciliopathy genes than patients with HLHS without developmental delay.

Methods: Ciliopathy gene variant burden was determined utilizing a summative C-score for 14 ciliopathy genes in children with HLHS $(n=24)$. Mean summative C-scores were compared between children with and without developmental delay. Genome-wide randomizing gene sets were evaluated as a scoring control.

Results: Children with developmental delay had a mean summative C-score of 4.05 in ciliopathy genes as compared to a mean summative C-score of 2.02 for children without developmental delay. This difference in means was higher than $99.1 \%$ (empirical $P$ value $<0.01)$ of 2 million random lists of 14 genes.

Conclusion: Genetically complex disorders such as ciliopathies can be assessed to determine phenotypic risk with summative C-score in appropriately chosen gene sets. If these results are replicated in subsequent cohorts, a diagnostic gene panel could identify risk for developmental delay and other ciliopathy-related comorbidities in infants with congenital heart disease.

Genet Med advance online publication 27 October 2016

Key Words: ciliopathy; CADD; developmental delay; hypoplastic left heart syndrome
Ciliary dysfunction is an increasingly appreciated developmental cause of congenital heart disease (CHD). ${ }^{1,2}$ Ciliary dysfunction has been described with $\mathrm{CHD}$ in the context of laterality defects such as heterotaxy for some time. ${ }^{3}$ Ciliary function is also critical in the developing embryo for neuronal migration. ${ }^{4-6}$ Ciliary dysfunction is known to cause a wide range of phenotypic disorders, including birth defects and developmental delay. ${ }^{7-9}$ It is for these reasons that we hypothesized that individuals with $\mathrm{CHD}$ due to ciliary dysfunction are at increased risk for developmental delay.

Disorders causing ciliary dysfunction are known to have multigenic inheritance. ${ }^{10}$ Multigenic inheritance is difficult to assess clinically. New standardized methods of assessing variants have made it possible to assess the burden of molecular disruption across a pathway. Combined annotation-dependent depletion (CADD) is a method that assigns a "C-score" to each variant, simplifying the level of molecular disruption into a standardized quantity. ${ }^{11}$

Our hypothesis is that children who have CHD due to ciliary dysfunction are at increased risk for developmental delay due to abnormal neuronal migration. To test this hypothesis, we evaluated a cohort of children with hypoplastic left heart syndrome and known developmental phenotype to assess the burden of variants in 14 genes associated with ciliary dysfunction. We were then able to determine whether there was a difference in the level of molecular disruption between groups with and without developmental delay.

\section{MATERIALS AND METHODS}

\section{Population}

All children had isolated hypoplastic left heart syndrome as defined by the Society of Thoracic Surgery diagnostic criteria. All children underwent developmental evaluation using the Bayley Scales of Infant Development-III (BSID-III) to evaluate cognitive, language, and motor development as well as wholeexome sequencing. Twenty-four children fit these criteria and were included in the analysis. No children were excluded from the study. Developmental delay was defined by an average cognitive and/or language composite score on the BSIDIII greater than 1 standard deviation below the mean (score $<85){ }^{12}$ The developmental trajectories by patient are shown in Supplementary Figure $\mathbf{S 1}$ online. At the time of their first BSID-III assessment, all subjects had undergone at least one open-heart procedure. Motor delay was not included because

The last two authors are the senior authors.

The first two authors contributed equally to this work

${ }^{1}$ Department of Pediatrics, Medical College of Wisconsin, Milwaukee, Wisconsin, USA; ${ }^{2}$ Department of Surgery, Medical College of Wisconsin, Milwaukee, Wisconsin, USA; ${ }^{3}$ Herma Heart Center, Children’s Hospital of Wisconsin, Milwaukee, Wisconsin, USA. Correspondence: Gabrielle C. Geddes (ggeddes@mcw.edu) 
the score could be influenced by surgical status. Groups were evaluated and there was no significant difference according to Fischer's exact test between groups for gender, prenatal diagnosis, need for extracorporeal membrane oxygenation (ECMO), maternal age, marital status, education level, selfreported race, socioeconomic status, or insurance type. As would be anticipated, Fischer's exact test showed that patients in the developmentally delayed group were significantly more likely to require tube feeding $(P>0.05)$ at initial discharge. ${ }^{12}$ This information is available in more detail in Supplementary Table S1 online. None of the children were premature $(<37$ weeks gestation), had extracardiac anomalies, or had a known genetic anomaly.

\section{Gene list}

The gene list utilized in our study comprises genes previously found to cause molecular disruption in children with congenital heart disease and ciliary dysfunction on videomicroscopy. This list includes CCDC39, CCDC40, DNAH5, DNAH11, DNAI1, DNAI2, DNAL1, KTU, LRRC50, OFD1, RPGR, RSPH4A, RSPH9, and TXNDC3.13

\section{Sequencing}

Exome sequencing was completed with whole-exome capture with an Agilent (Santa Clara, CA) SureSelect Human All Exon kit v.5 and complete next-generation sequencing with an Illumina (San Diego, CA) Hi-Seq 2000. Exome coverage information is available in Supplementary Table $\mathbf{2} 2$ online. The average read depth for variants included in the summative C-score was 58. We were able to confirm variants in all children with variants for whom RNASeq data was available for review. Exome capture sequence processing was performed using BWA and GEMINI. ${ }^{14,15}$

\section{CADD thresholds}

To determine the C-score to use as a clinically relevant cutoff, we compared CADD v1.2 to 12,000 sites with ClinVar annotations (database edition 20150305), which randomly selected 6,000 benign and 6,000 pathogenic variants. ${ }^{11}$ The C-score threshold of 4.07 was the point at which $50 \%$ of pathogenic variants were retained. At this threshold, approximately $3 \%$ of benign variants were retained. The C-score at this threshold was 4.07, as shown in Figure 1.

\section{Analysis}

All analyses were performed in R with plyr. ${ }^{16}$ All variants found in all genes in a given gene list with an individual C-score $>4.07$ are included in an individual's summative C-score, which is the sum of all $\mathrm{C}$-scores above the threshold within the gene list. Summative C-scores representing the burden of genetic variation in 14 candidate genes associated with cilia development and function were compared between the children with developmental delay and those without delay. The differences in mean summative $\mathrm{C}$-scores for each phenotypic group were compared between the gene list of interest and randomized lists. Specific variants included in the summative C-scores are listed in Supplementary Table S3 online.

\section{Statistical analysis}

Two million random lists of 14 genes were assembled and measured for summative C-score per patient and compared by phenotype. This creates a null distribution for the method genome-wide, such that a given gene list can be compared to blind selection. The result is a percentile (empirical $P$ value) for our ciliopathy gene list as compared to the rest of the genome sequenced. To further control our data, we utilized our method with 2 million sets of 24 individuals from the 1000 Genomes Project (edition 20130502, containing 2,504 individuals) set to determine the differences in summative $\mathrm{C}$-scores in the context of a healthy population across 14 genes. Sex chromosomes were excluded from this analysis.

\section{RESULTS}

\section{Patient population}

The children completed a total of 74 BSID-III evaluations. Mean age at BSID-III evaluation was 19.3 months. Sixty-two percent were male, and $62 \%$ were white non-Hispanic race/ethnicity by parental report. One-third of the children had other significant medical comorbidities in addition to their congenital heart disease. Mean age at first open-heart surgery was 11 days. Half of the children required supplemental tube feedings at the time of their first hospital discharge. Of the 24 children evaluated, 12 (50\%) had developmental delay and $12(50 \%)$ did not and performed in the average range. Of the 12 subjects with developmental delay, 8 (67\%) had delays in both cognitive and language development. Two (17\%) had delay in the cognitive domain only and two (17\%) in the language domain only. Developmental scores by patient are shown in Supplementary Figure $\mathbf{S 1}$ online.

The average (standard deviation) summative C-score for the ciliopathy gene list for children without delay was 2.02 (3.22); for children with delay, it was 4.05(4.57). This difference of 2.03 is greater than $99.1 \%$ (empirical $P$ value $<0.01$ ) of random gene lists, as shown in Figure 2. Investigation of genome-wide random gene sets of $N=14$ revealed that individuals with delay and without delay are equivalently burdened across the rest of the genome, 1.07 (2.34) and 0.98 (2.14), respectively.

Among the 1000 Genomes Project controls, genome-wide random gene sets have an equivalent score of 1.18 (1.18). The difference between groups of 12 individuals was remarkably normally distributed, with a mean of 0 and standard deviation of 1. A difference of 2.03 registers above the 97th percentile, indicating a gap of the magnitude seen between the developmentally delayed and the nondelayed is unlikely to occur by random chance $(P<0.04)$.

\section{DISCUSSION}

Patients with HLHS and developmental delay have a higher level of disruption in ciliary genes. This supports our hypothesis that children with CHD due to ciliary dysfunction may be at risk for other complications of ciliary dysfunction, such as 
CADD's clinvar discrimination

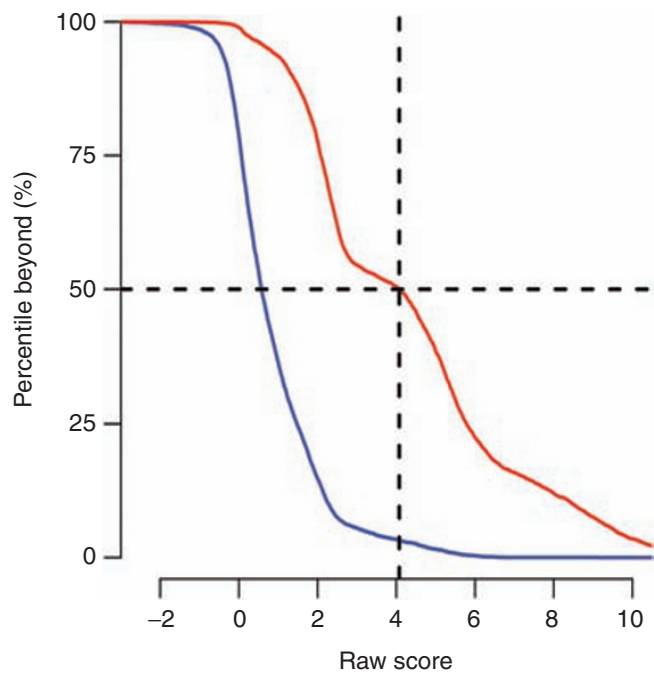

Threshold sweep ROC curve



Figure 1 C-score threshold. The graph on the left shows the discrimination ability of C-scores for variants annotated as either benign or pathogenic by ClinVar (random sample of 6,000 of each type of variant from the ClinVar database edition 20150305). Considering only variants with C-score above a threshold: proportion that retained benign variants in blue versus pathogenic in red. The dashed crossing lines indicate our threshold, 4.075 , chosen to capture $50 \%$ of pathogenic variants and minimal benign variants (3.2\%). The graph on the right shows the percentage of benign variants included versus the percentage of pathogenic variants included as the C-score threshold varies. The curve approaching the upper left indicates that combined annotation-dependent depletion is a good discriminator of pathogenic versus benign (area under curve $=89.7 \%$ ).

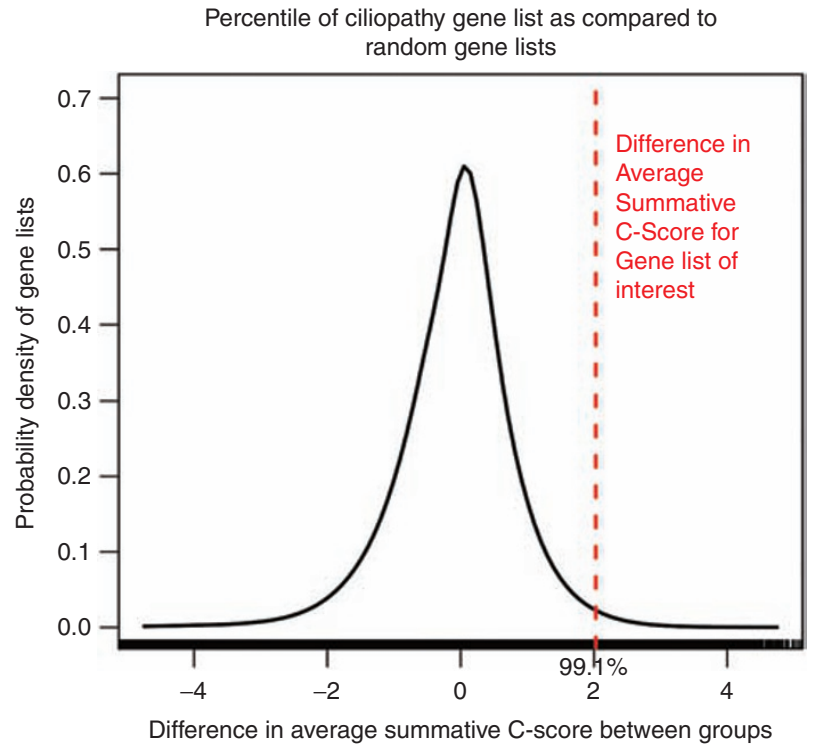

Figure 2 Percentile of ciliopathy gene list as compared to random gene lists. To estimate the significance of a C-score difference between our developmentally delayed and nondelayed patient groups, null permutation was performed by randomly selecting a gene list of equal size $(N=14)$ from the subject's exome data sets and computing the difference in average C-score 2 million times. The red line indicates that the ciliopathy gene list (with a difference between groups of 2.027) falls at the 99.14 percentile. Nearly one in five random gene lists had no difference in C-score. This symmetrical distribution indicates that the two patient groups have similar levels of variation across the genome.

developmental delay. The results demonstrate a novel method for assessing multigenic impacts on a discrete molecular pathway using summative $\mathrm{C}$-scores for a discrete gene list. The cohort showed a significant difference between summative
C-scores, with our list being at the 99.1 percentile of possible gene lists.

The sample size is a limiting factor in this study, and these results should be replicated in a larger cohort. Currently, the narrow difference and level of overlap in summative C-scores limit the ability to identify patients at risk for developmental delay using this method clinically. With larger sample sizes and more data, we will have the ability to create more specific cutoffs and define informative gene sets, furthering clinical utility. Another potential limitation of this study is that our analysis to standardize the summative C-score excluded sex chromosomes; however, our gene list included two genes (OFD1 and $R P G R$ ) on the $\mathrm{X}$ chromosome. We suspect that the exclusion of sex chromosomes did not impact our results because none of the variants contributing to the summative C-score (Supplementary Table S3 online) was in either of these genes.

The importance of developing methods to assess multigenic molecular pathways is clinically critical and more accessible given the availability of sequencing panels. Further investigation of these methods will eventually lead to clinical methods for determining the risk for disorders of complex inheritance such as developmental delay, which can lead to more aggressive early intervention.

\section{SUPPLEMENTARY MATERIAL}

Supplementary material is linked to the online version of the paper at http://www.nature.com/gim

\section{ACKNOWLEDGMENTS}

Although this project had no direct funding, we acknowledge that the exome data utilized in this project were initially funded by the Wanek Family and Wolfe Family to examine genetic causes of hypoplastic left heart syndrome. 


\section{BRIEF REPORT}

\section{DISCLOSURE}

K.S. is an employee of TAI Diagnostics, a commercial entity unrelated to this research. M.M. and A.T.M. are cofounders of TAl Diagnostics. The other authors declare no conflict of interest.

\section{REFERENCES}

1. Rao Damerla R, Gabriel GC, Li Y, et al. Role of cilia in structural birth defects: insights from ciliopathy mutant mouse models. Birth Defects Res C Embryo Today 2014;102:115-125.

2. Li Y, Klena NT, Gabriel GC, et al. Global genetic analysis in mice unveils central role for cilia in congenital heart disease. Nature 2015;521:520-524.

3. Sutherland MJ, Ware SM. Disorders of left-right asymmetry: heterotaxy and situs inversus. Am J Med Genet C Semin Med Genet 2009;151C:307-317.

4. Guemez-Gamboa A, Coufal NG, Gleeson JG. Primary cilia in the developing and mature brain. Neuron 2014;82:511-521

5. Métin C, Pedraza M. Cilia: traffic directors along the road of cortical development. Neuroscientist 2014;20:468-482.

6. Sarkisian MR, Guadiana SM. Influences of primary cilia on cortical morphogenesis and neuronal subtype maturation. Neuroscientist 2015;21:136-151.

7. Baker K, Northam GB, Chong WK, Banks T, Beales P, Baldeweg T. Neocortical and hippocampal volume loss in a human ciliopathy: A quantitative MRI study in Bardet-Biedl syndrome. Am J Med Genet A 2011;155A:1-8.
8. Brown JM, Witman GB. Cilia and Diseases. Bioscience 2014;64: 1126-1137.

9. Sattar S, Gleeson JG. The ciliopathies in neuronal development: a clinical approach to investigation of Joubert syndrome and Joubert syndrome-related disorders. Dev Med Child Neurol 2011;53:793-798.

10. Schäffer AA. Digenic inheritance in medical genetics. J Med Genet 2013;50:641-652.

11. Kircher M, Witten DM, Jain P, O'Roak BJ, Cooper GM, Shendure J. A general framework for estimating the relative pathogenicity of human genetic variants. Nat Genet 2014;46:310-315.

12. Mussatto KA, Hoffmann RG, Hoffman GM, et al. Risk and prevalence of developmental delay in young children with congenital heart disease. Pediatrics 2014;133:e570-e577.

13. Nakhleh N, Francis R, Giese RA, et al. High prevalence of respiratory ciliary dysfunction in congenital heart disease patients with heterotaxy. Circulation 2012;125:2232-2242.

14. Li H. Aligning sequence reads, clone sequences and assembly contigs with BWA-MEM. Quant Bio/ Genomics 2013. arXiv:1303.3997v1 [q-bio.GN]

15. Paila U, Chapman BA, Kirchner R, Quinlan AR. GEMINI: integrative exploration of genetic variation and genome annotations. PLoS Comput Biol 2013;9:e1003153.

16. Wickham, H. The split-apply-combine strategy for data analysis. I Stat Softw 2011;40:1-29. 